\title{
ERRATUM
}

\section{On a General Transformation Making a Dissimilarity Matrix Euclidean (DOI: 10.1007/s00357-007-0005-y)}

\author{
Jacques Bénasséni \\ Université de Haute Bretagne, France \\ Mohammed Bennani Dosse \\ Université de Haute Bretagne, France \\ Serge Joly \\ Université de Haute Bretagne, France
}

On page 42, Table 1 should be numbered Table 3, and Table 2 should be numbered Table 4. On page 43, Table 6 should be numbered Table 1 and ordered in first position among the seven tables, Table 7 should be numbered Table 2 and ordered in second position among the seven tables, Table 3 should be numbered Table 5, Table 4 should be numbered Table 6 , and Table 5 should be numbered Table 7 .

The Tables as numbered correctly follow.

Table 1. Dissimilarity matrix $d$ between 4 objects.

\begin{tabular}{|c|cccc|}
\hline & 1 & 2 & 3 & 4 \\
\hline 1 & 0 & & & \\
2 & 1 & 0 & & \\
3 & 1 & 4 & 0 & \\
4 & 0.05 & 0.2 & 9 & 0 \\
\hline
\end{tabular}


Table 2. Distance matrix $\varepsilon d$ derived from ACT.

\begin{tabular}{|c|cccc|}
\hline & 1 & 2 & 3 & 4 \\
\hline 1 & 0 & & & \\
2 & 2.6945 & 0 & & \\
3 & 2.6945 & 3.2275 & 0 & \\
4 & 2.6553 & 2.6568 & 4.9085 & 0 \\
\hline
\end{tabular}

Table 3. Distance matrix $\varepsilon d$ derived from $d^{0.1}$.

\begin{tabular}{|c|cccc|}
\hline & 1 & 2 & 3 & 4 \\
\hline 1 & 0 & & & \\
2 & 2.9393 & 0 & & \\
3 & 2.9393 & 3.5971 & 0 & \\
4 & 2.1652 & 2.4880 & 4.6622 & 0 \\
\hline
\end{tabular}

Table 4. Distance matrix $\varepsilon d$ derived from $1-e^{-7 d}$.

\begin{tabular}{|c|cccc|}
\hline & 1 & 2 & 3 & 4 \\
\hline 1 & 0 & & & \\
2 & 3.2722 & 0 & & \\
3 & 3.2722 & 3.2984 & 0 & \\
4 & 0.9668 & 2.4665 & 3.3968 & 0 \\
\hline
\end{tabular}

Table 5. Distance matrix $\varepsilon d$ derived from the subdominant ultrametric.

\begin{tabular}{|c|cccc|}
\hline & 1 & 2 & 3 & 4 \\
\hline 1 & 0 & & & \\
2 & 0.9331 & 0 & & \\
3 & 4.5972 & 4.6400 & 0 & \\
4 & 0.2299 & 0.9194 & 4.8209 & 0 \\
\hline
\end{tabular}

Table 6. Distance matrix derived from minimisation of STRESS

\begin{tabular}{|c|cccc|}
\hline & 1 & 2 & 3 & 4 \\
\hline 1 & 0 & & & \\
2 & 1.2125 & 0 & & \\
3 & 3.4875 & 4.7000 & 0 & \\
4 & 2.3250 & 1.1125 & 5.8125 & 0 \\
\hline
\end{tabular}

Table 7. Distance matrix derived from minimisation of SSTRESS

\begin{tabular}{|c|cccc|}
\hline & 1 & 2 & 3 & 4 \\
\hline 1 & 0 & & & \\
2 & 0.7213 & 0 & & \\
3 & 4.1635 & 4.8849 & 0 & \\
4 & 4.0890 & 3.3677 & 8.2526 & 0 \\
\hline
\end{tabular}

